\title{
First report of tomato chlorosis virus in pepper in Greece
}

\author{
Vasileia Gavrili ${ }^{1}$. Leonidas Lotos ${ }^{1} \cdot$ Nikolaos Katis $^{1} \cdot$ Varvara Maliogka $^{1}$ (C) \\ Received: 4 November 2021 / Accepted: 22 November 2021 / Published online: 29 November 2021 \\ (c) Società Italiana di Patologia Vegetale (S.I.Pa.V.) 2021
}

Keywords Pepper yellowing $\cdot$ Crinivirus $\cdot$ Tomato chlorosis virus

In February 2021, pepper plants displaying symptoms of pepper yellows disease (PYD) such as interveinal yellowing, upward leaf-rolling, short internodes and fruit malformation were observed in several fields in Tympaki (Crete), Greece. To determine putative associated pathogen(s), samples were collected from 25 symptomatic ( 13 from field PeTi, 12 from field $\mathrm{PeKu}$ ) and 10 asymptomatic (5/field) pepper plants. Total RNA (White et al. 2008) was extracted from two composite samples (PeTiA and PeKuB) containing the symptomatic plants of each field and were characterized by high-throughput sequencing analysis on a NovaSeq 6000 platform (Illumina, Inc., Macrogen). The HTS run yielded approximately 50 million $101 \mathrm{nt}$ paired-end reads for each sample. De novo assembly produced 1,324 contigs and 901 contigs for PeTiA and PeKuB, respectively. BLASTn revealed the presence of two contigs in each sample which shared $>99 \%$ nucleotide identities with tomato chlorosis virus RNA1 and RNA2 sequence deposited in GenBank. To confirm HTS results, all symptomatic individual plants were subjected to real time RT-PCR assay utilizing the protocol described by Papayannis and colleagues (2011). ToCV was identified in all plants of the field PeTi and in four plants from the field PeKu. The remaining eight samples of the field PeKu were positive for polerovirus infection using the RT-PCR protocol developed by Lotos and colleagues (2017). Furthermore, all positive samples in ToCV infection were subjected to RT-PCR assay using the primer pair $\mathrm{p} 22 \mathrm{~F}\left(5^{\prime}-\right.$ AGTATGGATCTCACTGGTT-3')/p22R(5'AGCAAAACG ATCACCCAT-3'), to amplify a fragment of $603 \mathrm{bp}$. Two samples were selected for Sanger sequencing and BLASTn analysis revealed that Greek isolates PeTi8 (OK149170) and

Varvara Maliogka

vmaliogk@agro.auth.gr

1 Laboratory of Plant Pathology, School of Agriculture, Aristotle University of Thessaloniki, 54124 Thessaloniki, Greece
PeKu51 (OK149169) were similar to the HS isolate from Korea (KP137098.1) with >99\% nt identity. To the best of our knowledge, this is the first report of ToCV in pepper in Greece, thus further increasing the number of viruses associated with PYD in the country.

Funding The research project was supported by the Hellenic Foundation for Research and Innovation (H.F.R.I.) under the "1st call for H.F.R.I. Research Projects to support Faculty Members \& Researchers and the Procurement of high-cost research equipment grant (Project Number: 3719).

\section{Declarations}

Conflict of interest The authors have no conflicts of interest to declare that are relevant to the content of this article.

\section{References}

Lotos L, Olmos A, Orfanidou C, Efthimiou K, Avgelis A, Katis NI, Maliogka VI (2017) Insights Into the Etiology of Polerovirus Induced Pepper Yellows Disease. Phytopathology 107(12):15671576. https://doi.org/10.1094/PHYTO-07-16-0254-R

Papayiannis LC, Harkou IS, Markou YM, Demetriou CN, Katis NI (2011) Rapid discrimination of Tomato chlorosis virus, Tomato infectious chlorosis virus and co-amplification of plant internal control using real-time RT-PCR. J Virol Methods 176:53-59. https://doi.org/10.1016/j.jviromet.2011.05.036

White EJ, Venter M, Hiten NF, Burger JT (2008) Modified cetyltrimethylammonium bromide method improves robustness and versatility: the benchmark for plant RNA extraction. Biotechnol J 3(11):1424-1428. https://doi.org/10.1002/biot.200800207

Publisher's Note Springer Nature remains neutral with regard to jurisdictional claims in published maps and institutional affiliations. 